\title{
DEPENDENCE OF THE SNS TRANSFER LINES AND ACCUMULATOR RING ON LINAC ENERGY*
}

\author{
D. Raparia, Y. Y. Lee, J. Wei and W. T. Weng, BNL, Upton, NY 11972,USA
}

\begin{abstract}
One of the options considered for the SNS linac, to reduce the cost, was to lower the energy to $840 \mathrm{MeV}$ and leave space in the tunnel for a future upgrade to $1.3 \mathrm{GeV}$ either by adding cryo-modules or increasing the gradient in the SC linac. A linac energy other than $1.0 \mathrm{GeV}$ will have an impact on the transfer line and accumulator ring. The energy impacts the location of the corrector cavity in the HEBT, the injection magnet, beam power, $\mathrm{dE} / \mathrm{dx}$, multiple scattering and $\mathrm{H}$ stripping, neutron production at the target etc. These issues will discuss, and changes required in the transfer lines and accumulator ring to accommodate lower energy beam are presented.
\end{abstract}

\section{INTRODUCTION}

The SNS accelerator complex includes a $1.0 \mathrm{GeV}$ Superconducting (SC) linac[1], High Energy Beam Transport (HEBT), accumulator ring, and Ring to Target Beam Transport (RTBT)[2,3]. For the SC linac, one cavity per klystron was chosen to achieve high quality and reliable beam. This option increased the cost of linac. To fit the cost within the budget, one of the options considered was to lower the linac energy to about 840 $\mathrm{MeV}$. The proposal is to leave the space in the tunnel for future to go to $1.0 \mathrm{GeV}$. A linac energy other than 1.0 $\mathrm{GeV}$ will have the following impact on the transfer line and accumulator ring. (1) location of the Energy Corrector Cavity (ECC) in the HEBT, (2) injection magnet, (3) beam power, (4) RF frequency, and RF system, (5) issues related to $\mathrm{dE} / \mathrm{dx}$, multiple scattering, and $\mathrm{H}$ minus stripping, and (6) neutron production at the target.

\section{LOCATION OF THE ECC IN THE HEBT}

Due to amplitude and phase control error in the linac, the beam will suffer from energy jitter. This energy jitter will result in there being beam in the extraction beam gap in the accumulator ring, which will be lost during the rise time of the extraction kickers. To correct the energy jitter from the linac, there is ECC in the HEBT whose phase is locked with the last linac cavity such that the synchronous particle arrives at the cavity at zero phase and does not lose or gain the energy. The lower/higher energy particle arrives at the cavity late/early and gain/lose the energy accordingly [4]. If the linac energy is lowered this phase slip will increase due to length change and the energy change. This phase slip $\Delta \phi_{\mathrm{L}}$ is given by

$$
\Delta \phi_{L} \equiv \frac{1}{\gamma(\gamma+1)} \frac{\Delta T}{T} \frac{L}{\beta c} 2 \pi f
$$

\footnotetext{
* Work performed under the auspices of the US Department of Energy.
}

where $\beta, \gamma$ are the relativistic parameters, $\mathrm{T}$ is the design energy and $\Delta \mathrm{T}$ is the energy deviation from the design energy, $\mathrm{L}$ is the length in which phase slip occurs and $\mathrm{f}$ is the frequency. Figure 1 shows the phase slip per $\mathrm{MeV}$ as a function of distance.

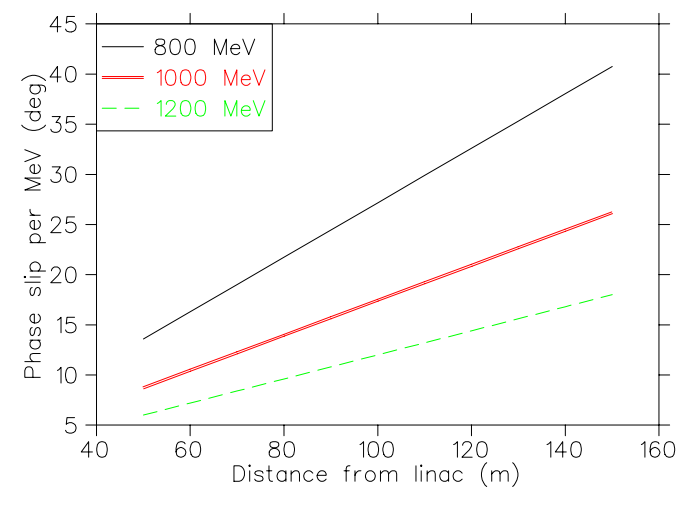

Figure 1: Phase slip per $\mathrm{MeV}$ as a function of distance between the last linac cavity and energy corrector cavity.

\section{INJECTION CHICANE MAGNET}

The $1 \mathrm{~ms}$ long linac beam pulse will be injected into the accumulator ring via charge exchange injection in about 1200 turns[5]. To reduce the foil traversals and space charge density, linac beam with very low emittance $(0.28$ pi $\mathrm{mm} \mathrm{mrad}, \mathrm{rms}$ ) is painted into to a very high emittance area of 120-160 pi mm mrad[6]. This is accomplished by moving the closed orbit at the charge exchange foil using a chicane made of four vertical dipoles and four horizontal dipoles. The chicane magnet at the foil has following constraints. (1) the field in this magnet should be such that the Lorenz stripping of $\mathrm{H}^{-}$should be acceptable with the loss criteria, and (2) the field should also prevent foil stripping to $\mathrm{H}^{0}$ in $\mathrm{n}=4$ and lower exited states. As pointed out in reference 7 due the quantum nature of excited state of $\mathrm{H}^{0}$, if the energy difference is more than $\pm 5 \%$, two injection chicane magnets have to change. Table I shows the required magnetic field for different injection energies.

Table I: Required magnetic field for the middle chicane magnet

\begin{tabular}{|c|c|}
\hline Injection Energy $(\mathrm{MeV})$ & $\begin{array}{c}\text { Magnetic field in middle } \\
\text { chicane magnet }(\mathrm{T})\end{array}$ \\
\hline 800 & 0.29 \\
\hline 900 & 0.27 \\
\hline 1000 & 0.25 \\
\hline 1100 & 0.23 \\
\hline 1200 & 0.215 \\
\hline 1300 & 0.205 \\
\hline
\end{tabular}




\section{BEAM POWER}

The space charge tune shift in the accumulator ring is proportional to current and inversely proportional to $\beta^{2} \gamma^{3}$.

$$
\Delta v \propto \frac{I}{\beta^{2} \gamma^{3}}
$$

In order to keep the same space charge tune shift, beam current has to be lower for lower linac energy. Figure 2 shows the beam power as a function of linac energy for a given space charge tune shift (0.15).

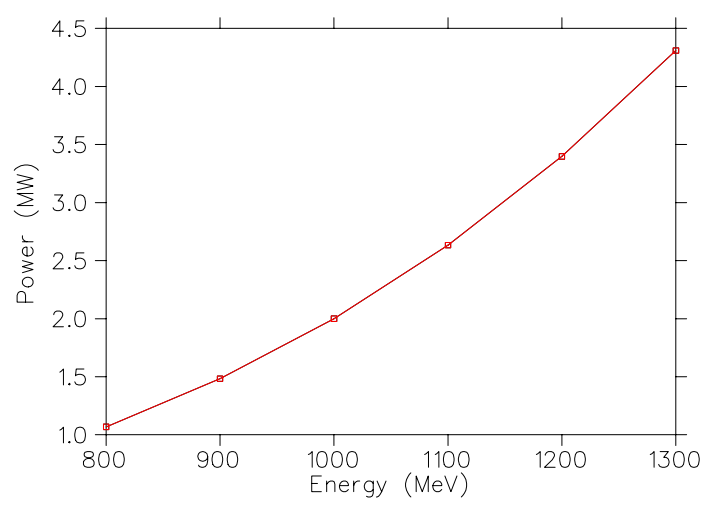

Figure 2: Beam power as function of injection energy in the accumulator ring for space charge tune shift of 0.15 .

\section{RF FREQUENCY}

The beam revolution frequency will be lower for lower linac energy, so the RF frequency in the accumulator ring must also be lower. This might require tuning the power supply. No change in the ferrite is required. With $\beta=0.875$ at $1 \mathrm{GeV}$ and $\beta=0.842$ at $800 \mathrm{MeV}, 10 \mathrm{kV}$ per gap at $1 \mathrm{GeV}$ reduces to $9.6 \mathrm{kV}$ per gap at $800 \mathrm{MeV}$ and should be no problem.

\section{ENERGY STRAGGLING, MULTIPLE SCATTERING AND H' STRIPPING DUE TO RESIDUAL GAS}

In the transfer line and accumulator ring the beam traverses material at two places. First during the injection process (charge exchange injection) beam passes through the $300 \mu \mathrm{g} / \mathrm{cm}^{2}$ thick carbon foil and suffers energy straggling and multiple scattering. The other time is when beam passes through a $4 \mathrm{~mm}$ thick inconel window in the RTBT about 2 meters in front of target.

The mean rate of energy loss is given by Bethe-Bloch equation

$$
-\frac{d E}{d x}=K z \frac{Z}{A} \frac{1}{\beta^{2}}\left[\frac{1}{2} \ln \frac{2 m_{e} c^{2} \beta^{2} \gamma^{2} T_{M A X}}{I^{2}}-\beta^{2}-\frac{\delta}{2}\right]
$$

$$
T_{\max }=\frac{2 m_{e} c^{2} \beta^{2} \gamma^{2}}{1+2 \gamma m_{e} / M+\left(m_{e} / M\right)^{2}}
$$

Here $T_{\text {MAX }}$ is the maximum kinetic energy which can be imparted to a free electron in a single collision. $\mathrm{K} / \mathrm{A}=0.307075 \mathrm{MeV} \mathrm{g}^{-1} \mathrm{~cm}^{2}, \mathrm{Z}$ is the atomic number of the media, $\mathrm{m}_{\mathrm{e}}$ is the mass of the electron, $\mathrm{I}$ is the mean excitation energy, $\delta$ is density effect correction to the ionization energy loss, $c$ is velocity of light and $\beta, \gamma$ are the relativistic parameters. The multiple scattering angle is given by

$\theta_{0}=\frac{13.6 \mathrm{MeV}}{\beta c p} z \sqrt{\frac{x}{X_{0}}}\left[1+0.038 \ln \left(\frac{x}{X_{0}}\right)\right]$

Here $\mathrm{p}, \beta \mathrm{c}$ and $\mathrm{z}$ are the momentum, velocity, and charge number of the incident particle, and $\mathrm{x} / \mathrm{X}_{0}$ is the thickness of the scattering medium in radiation lengths. Figures 3 shows the energy losses due to this processes as a function of the energy. Figure 4 shows the multiple scattering angles as a function of energy.

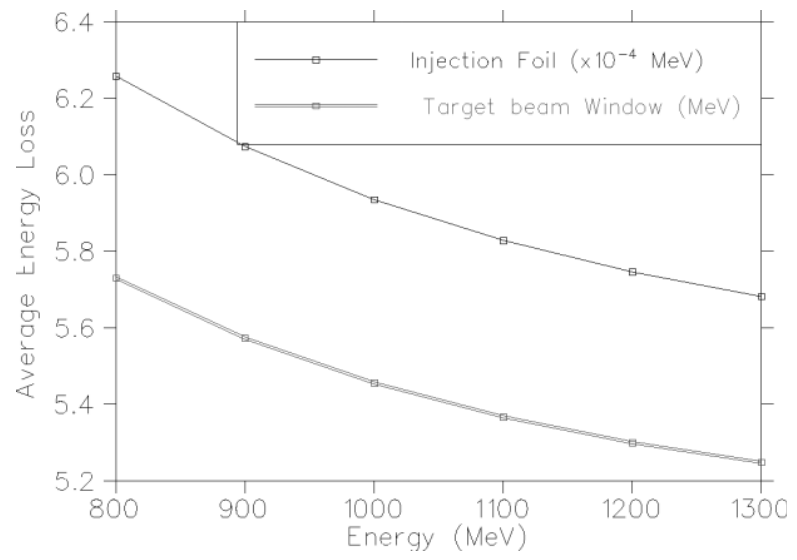

Figure 3: Average energy loss as a function of energy for proton beam window in RTBT which is $4 \mathrm{~mm}$ thick made of inconel and injection carbon foil $300 \mu \mathrm{g} / \mathrm{cm}^{2}$.

$\mathrm{H}$ minus gas stripping will also be affected by lower energy. At $1 \mathrm{GeV}$ for residual gas consisting of $40 \% \mathrm{H}_{2}$, $40 \% \mathrm{H}_{2} \mathrm{O}$, and $20 \% \mathrm{CO}$ will have stripping cross section < $1 \times 10^{-18} \mathrm{~cm}^{2}$ and corresponding stripping losses at $5 \times 10^{-8}$ Torr, will be $0.3 \mathrm{nA} / \mathrm{m}$. For $800 \mathrm{MeV}$ this loss will increase by $8 \%$ since the stripping cross section varies as $1 / \beta^{2}$. 


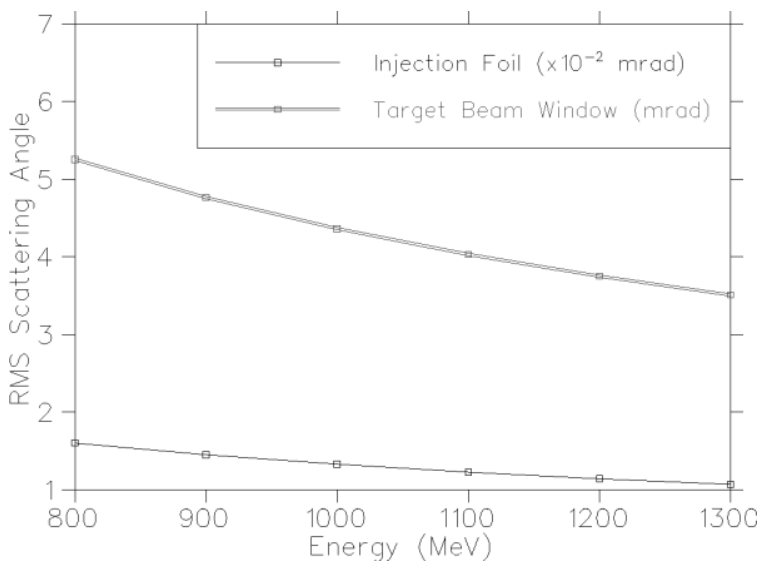

Figure 4: RMS multiple scattering angles as a function of energy for proton beam window in RTBT which is 4 $\mathrm{mm}$ thick made of inconel and injection carbon foil $300 \mu \mathrm{g} / \mathrm{cm}^{2}$.

\section{NEUTRON PRODUCTION AT THE TARGET}

Neutron production at the target is proportional to the proton beam power; therefore neutron production at 1 MW ( $800 \mathrm{MeV}$, see figure 1) will be about half that of 1 $\mathrm{GeV}$ protons. Figure 5 shows neutron yield per MW of beam power as a function of proton energy on a lead target[8]. Below $1 \mathrm{GeV}$ the curve decreases rapidly.

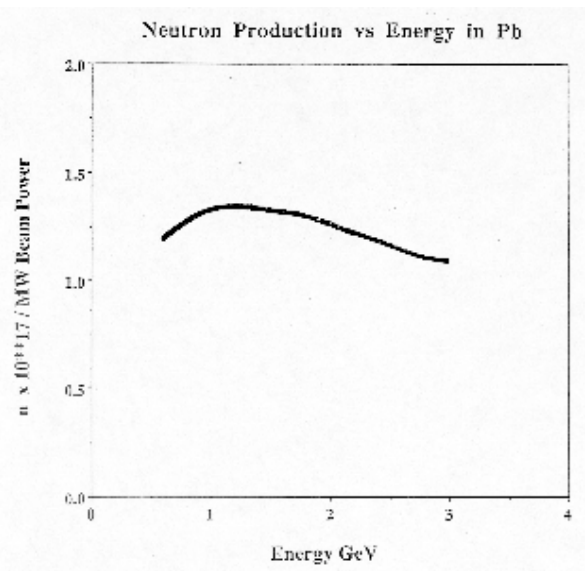

Figure 5: Neutron yield per MW of beam power for a lead target.

\section{REFERENCES}

[1] J. Stovall, et al., "Expected Beam Performance of the SNS Linac", This Conference.

[2] W. T. Weng, et al., "The design of the SNS Ring and Transport System", This Conference.

[3] J. Wei, et al., Performance, Optimization and Path

Towards a 2 MW Spallation Neutron Source", This Conference.

[4] D. Raparia, et al., "Impact of a Lower Energy Linac on Transfer Lines and accumulator Ring", SNS/BNL design note, May 20, 2000.

[5] J. Wei, et al., Choice of the Injection Scenario for the Spallation Neutron Source Ring", This conference. [6] J. Beebe Wang, et al., "Injection Stripping Foil issues in the SNS Accumulator Ring", This Conference.

[7] D. Raparia, et al., "Impact of the super conducting linac on HEBT and the ring", SNS/BNL design note, January 14, 2000

[8] H. Ludewig , private communication. 